\title{
Neurosurgical transection of the breast: an unexpected extracranial complication of ventriculoperitoneal shunt insertion
}

\author{
Molly Jakeman, MBChB, MRCS, ${ }^{1}$ Ranjeet Jeevan, MA (Cantab), MBBChir, MRCS, ${ }^{1}$ \\ Sasha C. Burn, MBChB, FRCS(SN), ${ }^{2}$ and Sian Falder, MBBS, FRCS(Plast) ${ }^{1}$ \\ ${ }^{1}$ Regional Burns and Paediatric Plastic Surgery Service, and 2Department of Neurosurgery, Alder Hey Children's NHS \\ Foundation Trust, Liverpool, United Kingdom
}

\begin{abstract}
Ventriculoperitoneal (VP) shunt placement is among the most common surgical procedures undertaken by neurosurgeons. Complications arising from the thoracic portion of the shunt are relatively rare in comparison with those of the intraventricular and peritoneal portions. Disruption of primary breast development following VP shunt placement has not previously been reported. The authors describe the case of a 15 -year-old girl referred to the plastic surgery department with a significant right breast deformity and associated asymmetry following VP shunt placement performed during the neonatal period. The calcified shunt was excised and the breast deformity was corrected surgically through multiple scar tissue releases and restoration of the normal breast parenchymal anatomy via a minimally invasive approach, resulting in an excellent aesthetic outcome.
\end{abstract}

This case highlights the potential for injury to occult breast tissue in pediatric patients undergoing VP shunt placement, which can impair subsequent cosmesis and quality of life.

https://thejns.org/doi/abs/10.3171/2017.7.PEDS16708

KEY WORDS hydrocephalus; ventriculoperitoneal shunt; CSF shunt; breast development; breast reconstruction

$\mathrm{B}$ REAST development begins in utero, with a well-defined primary breast bud formed by the end of the first trimester. Breast tissue development begins in the second trimester, and the nipple is formed during the third trimester. ${ }^{3}$ At birth, the volume of breast tissue present is variable, but there is no significant difference between that in males and females. The breast tissue is quiescent until puberty when further stromal and ductal development occurs in response to rising estrogen levels. The macroscopic 5 stages of breast development were described by Tanner in $1962.5,9$

Ventriculoperitoneal (VP) shunt placement and revision are among the most common surgical procedures performed by neurosurgeons, and complications relating to the thoracic portion of the shunt are relatively rare compared with those of its other components. ${ }^{4,8}$ Injury to the developing breast in pediatric surgical procedures involving thoracostomy or thoracotomy is well described..$^{1,2,6} \mathrm{We}$ detail a previously unreported complication of primary breast development disruption secondary to insertion of a VP shunt in the neonatal period of a female patient.

\section{Case Report}

A 15-year-old girl was referred to our regional pediatric plastic surgery service for consideration of unilateral breast reconstruction.

She had been born prematurely, at just 25 weeks' gestation, and during her initial stay in our neonatal intensive care unit, she was found to have an intraventricular bleed. Posthemorrhagic hydrocephalus developed and a rightsided VP shunt was inserted at 4 weeks of age. The shunt remained patent for 12 years before requiring revision. A second VP shunt was placed on the left side to treat her hydrocephalus. The original shunt was left in place and, prior to presentation, had been asymptomatic. Both shunts used were barium-infused devices.

On presentation to our service 3 years later, significant

ABBREVIATIONS VP = ventriculoperitoneal.

SUBMITTED December 30, 2016. ACCEPTED July 12, 2017.

INCLUDE WHEN CITING Published online October 6, 2017; DOI: 10.3171/2017.7.PEDS16708. 
breast asymmetry was immediately apparent. The right breast was smaller than the left, sitting high and lateral when compared with the left breast and with deficient tissue in the inferomedial aspect. The breast was completely transected in a craniocaudal direction by the calcified and scarred nonfunctional VP shunt that had been placed in the neonatal period. In contrast, the left breast had a normal shape and position (Fig. 1). The patient's other medical history included cerebral palsy with diplegia for which she had undergone dorsal rhizotomy and numerous orthopedic procedures to improve the alignment of lower limbs. She had been treated for epilepsy with carbamazepine until the age of 6 . She mobilized with a frame (walker) and was noted to have mild learning difficulties. She had undergone mainstream schooling and was keen to progress to college-level education. She had become more aware and self-conscious of her breast asymmetry during her teenage years and wished to improve the aesthetic appearance of her right breast.

We referred her to our psychology service for initial assessment and counseling and discussed her case with the referring neurosurgical team. She was reviewed in our clinic on several occasions for discussion of possible treatment options, including removal of the VP shunt and excision of its associated scar tissue, with or without subsequent tissue expansion, and breast reconstruction with an implant or autologous tissue transfer technique.

The patient chose to undergo a combined operation in which the neurosurgeons excised both the cranial and right breast mound segments of the VP shunt and its surrounding scar tissue. For the breast, we used an inframammary approach after inducing general anesthesia. The shunt coursed medial to the nipple-areolar complex and was found to be surrounded by a heavily calcified capsule along its entire length. The shunt placement created an apparent cleft in the breast with normal breast parenchymal tissue found both lateral and, to a lesser extent, medial to the shunt. The total volume of breast tissue appeared smaller than that on the left side. We dissected superiorly to excise the shunt from the breast tissue together with the calcified capsule. Removal of this portion of the shunt and surrounding scar tissue resulted in an immediate improvement in the breast position and shape, indicating that scar tethering was responsible for the majority of asymmetry. However, the cleft in the breast tissue remained, and we incised some ligaments within the parenchyma to unfurl the remaining breast tissue. Medial and lateral portions of the breast tissue were sutured together to recreate a natural, albeit slightly smaller, breast. The cranial portion of shunt was excised, but a portion of shunt remained in the upper chest and supraclavicular regions. This segment was left in situ to reduce the amount of cutaneous scarring and because it had not been associated with any of the patient's concern. The patient tolerated this procedure well, no drains were required, and following an overnight stay, she was discharged with a dressing clinic appointment at 1 week. On follow-up, her wounds had healed well with a notable improvement in her breast symmetry.

However, the patient felt that the remaining segment of the shunt running from the cranium to the superior pole of the right breast was now noticeable. This was due to both the firmness of the calcification and the resulting soft-tissue distortion and pulling sensation on turning her neck. She expressed her wish to have this section removed and subsequently was admitted overnight for a second procedure to excise the remaining $23-\mathrm{cm}$ segment of shunt and associated scar tissue. We used a minimal access approach, with excision undertaken via three $2-\mathrm{cm}$ transverse incisions along the craniocaudal axis of the shunt to optimize the aesthetic outcome. Again, this procedure was well tolerated and required no drains.

She was delighted with the outcomes of her two surgeries and does not currently wish to undergo further reconstructive procedures. Her postoperative photographs at 10 months are shown in Fig. 2.

\section{Discussion}

VP shunt placement is the standard and definitive treatment of hydrocephalus in both pediatric and adult populations. ${ }^{7,8}$ The rate of shunt failure is thought to be as high as $40 \%$ at 1 year postoperatively; as a result, VP shunt placements and revisions are among the most common surgical procedures performed by neurosurgeons. ${ }^{4,8}$

Complications relating to the thoracic portion of the shunt are relatively rare in comparison with those associated with the intraventricular and peritoneal segments. ${ }^{8}$ Some of these complications have been described in female adult patients, including a CSF leak into the breast area with pseudocyst formation or CSF "galactorrhea," breast implant disruption in those with a previous breast augmentation, and even shunt obstruction by invasive breast carcinoma ${ }^{7,8}$ However, to our knowledge, disruption of primary breast development secondary to VP shunt

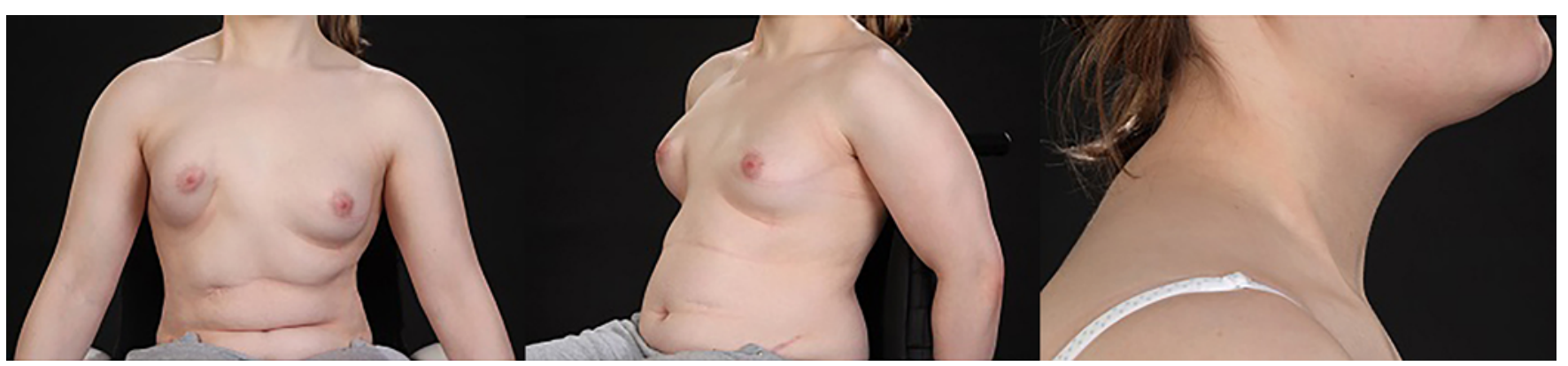

FIG. 1. Preoperative images showing breast asymmetry and heavily calcified shunt in the chest and neck areas. Figure is available in color online only. 


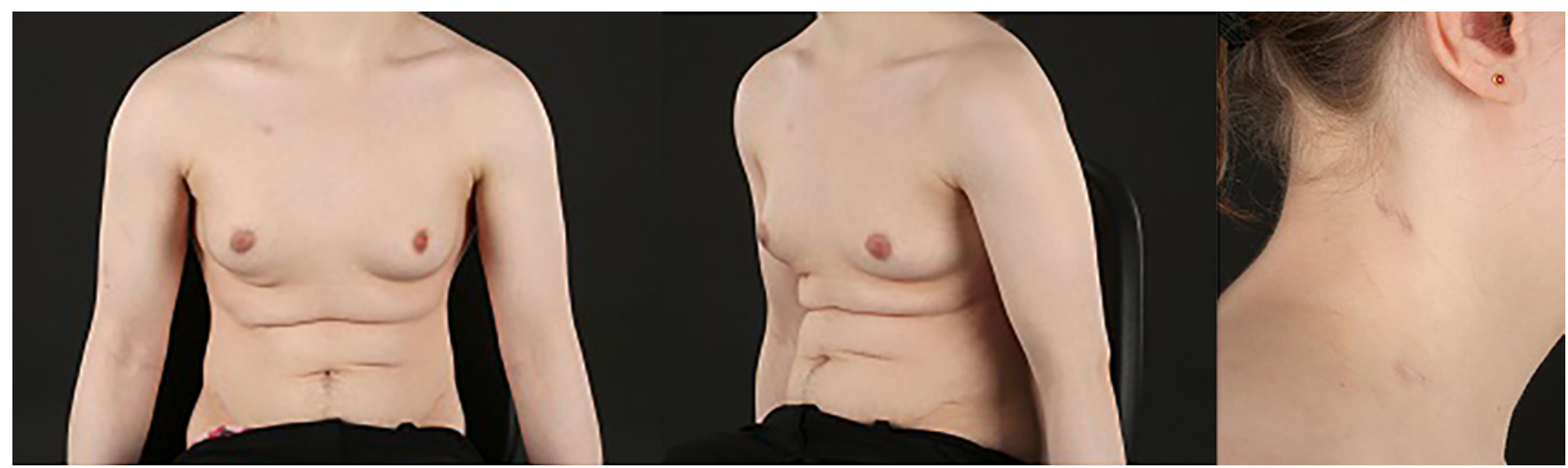

FIG. 2. Final postoperative images showing improved symmetry and contouring. Figure is available in color online only.

placement has not previously been described in the literature.

VP shunt placement through the soft tissue of the thoracic region is undertaken using a long trocar, and care must be taken to insert this using a thoracic course that avoids the breast area. While the appropriate axis for placement may be more apparent in the adult female patient with a developed breast, or even in those women with breast implants, it has not prevented iatrogenic injuries in the past. ${ }^{7,8}$ It is important to avoid injuring undeveloped and essentially occult breast tissue in the pediatric patient, and to remember that this tissue, and any associated deformity, will not become prominent until the onset of puberty. As puberty may occur early or late in patients with hydrocephalus, depending on its etiology, appropriate follow-up arrangements should be made to ensure that clinicians are able to identify and address a problem that may arise many years after the primary surgery. ${ }^{5}$

Our patient had a smaller volume of breast tissue on the right side than the left. It is unclear whether this was a natural variation between the two sides, or if it represented incomplete development of breast tissue due to the disruption caused by shunt placement. As breast tissue was present on either side of the shunt, it appears that the shunt must have been placed through the undeveloped infant breast. Although disruption of breast development following VP shunt placement has not previously been described, iatrogenic breast bud injury leading to breast hypoplasia is a recognized complication of other pediatric surgical interventions that involve thoracostomies or thoracotomies. ${ }^{1,2,6}$

Our unit uses barium-infused shunts, and there is much informal debate regarding the amount of barium present in the tubing and the resulting degree of calcification. It would seem logical that a smaller amount of barium present in the device would result in less surrounding calcification. However, the current focus in shunt tubing development is on reduction of infection rather than on calcification.

The authors of a cadaveric study have indicated that occult breast tissue in infants can extend as far as $1.5 \mathrm{~cm}$ peripheral to the areola margin. ${ }^{1}$ In neonates, as in adult women, breast tissue is delineated anteriorly and posteriorly by two layers of superficial fascia, which are connected by Cooper's ligaments. The Chassaignac bursa (retromammary space or submammary serous bursa) lies between the deeper layer of this superficial fascia and the underlying pectoral fascia. ${ }^{1}$ Therefore, shunt placement 2 $\mathrm{cm}$ medial or lateral to the areola margin and within the Chassaignac bursa is unlikely to disrupt breast development, and surgeons should refine their approach accordingly (Fig. 3).

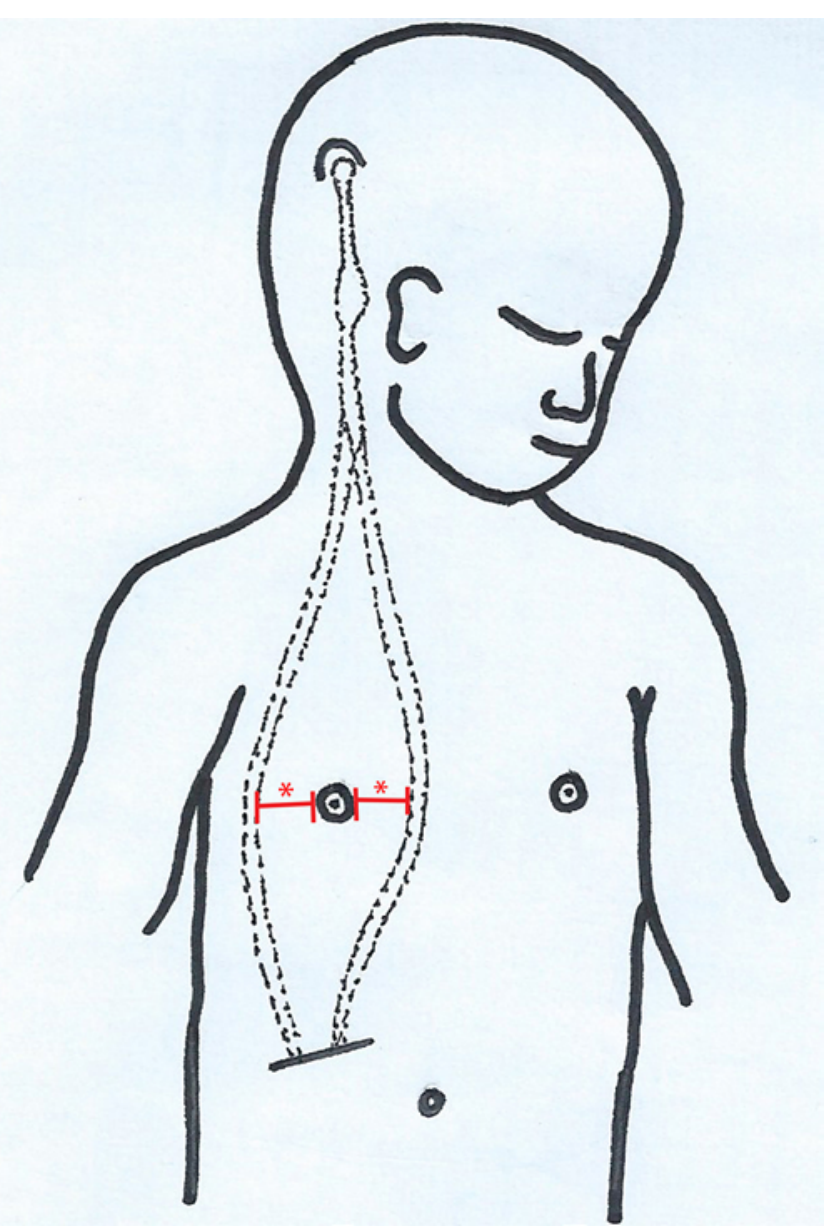

FIG. 3. Recommended positioning of the thoracic portion of VP shunts, either $2 \mathrm{~cm}$ medial or lateral to the areolar margin. Asterisk indicates $2 \mathrm{~cm}$. Figure is available in color online only. 
While the impact on breast mound development may be less evident in male patients, inadvertent placement of the shunt within the breast bud can still distort the chest wall significantly. In both male and female patients, the scarring and calcification associated with shunt placement may lead to aesthetic concerns as well as chronic pain and discomfort.

In conclusion, misplacement of a VP shunt in a pediatric patient can disrupt breast development, lead to pain and discomfort, and significantly affect the individual's subsequent physical and psychological well-being and quality of life. Neurosurgeons should be aware of this risk and take care to avoid injury to the breast bud during shunt placement. If this complication arises, they should consider early referral to an appropriate plastic surgery service to ensure optimal multidisciplinary management that is likely to involve a joint procedure to remove the shunt and its associated scar tissue along with suitable reconstructive techniques.

\section{References}

1. Cherup LL, Siewers RD, Futrell JW: Breast and pectoral muscle maldevelopment after anterolateral and posterolateral thoracotomies in children. Ann Thorac Surg 41:492-497, 1986

2. Goyal A, Mansel RE: Iatrogenic injury to the breast bud causing breast hypoplasia. Postgrad Med J 79:235-236, 2003

3. Javed A, Lteif A: Development of the human breast. Semin Plast Surg 27:5-12, 2013

4. Kulkarni AV, Riva-Cambrin J, Butler J, Browd SR, Drake JM, Holubkov R, et al: Outcomes of CSF shunting in children: comparison of Hydrocephalus Clinical Research Network cohort with historical controls: clinical article. J Neurosurg Pediatr 12:334-338, 2013
5. Marshall WA, Tanner JM: Variations in pattern of pubertal changes in girls. Arch Dis Child 44:291-303, 1969

6. Rainer C, Gardetto A, Frühwirth M, Trawöger R, Meirer R, Fritsch $\mathrm{H}$, et al: Breast deformity in adolescence as a result of pneumothorax drainage during neonatal intensive care. Pediatrics 111:80-86, 2003

7. Rudolph R, Burdick A: Silicone breast implant skewered by a ventriculoperitoneal shunt with secondary cerebrospinal fluid pseudocyst. Aesthet Surg J 35:NP179-NP181, 2015

8. Schrot RJ, Ramos-Boudreau C, Boggan JE: Breast-related CSF shunt complications: literature review with illustrative case. Breast J 18:479-483, 2012

9. Tanner JM: Growth at Adolescence. Oxford: Blackwell Scientific, 1962

\section{Disclosures}

The authors report no conflict of interest concerning the materials or methods used in this study or the findings specified in this paper.

\section{Author Contributions}

Conception and design: Jakeman, Jeevan. Acquisition of data: Jakeman, Jeevan. Analysis and interpretation of data: Jakeman, Jeevan. Drafting the article: Jakeman. Critically revising the article: Jeevan, Burn, Falder. Reviewed submitted version of manuscript: Burn, Falder. Approved the final version of the manuscript on behalf of all authors: Jakeman. Administrative/technical/material support: Jakeman.

\section{Correspondence}

Molly Jakeman, Regional Burns and Paediatric Plastic Surgery Service, Alder Hey Children's NHS Foundation Trust, Eaton Rd., Liverpool L12 2AP, United Kingdom. email: molly.jakeman@ doctors.org.uk. 